\title{
Generalizations of Integral Inequalities Similar to Hardy's Inequality
}

\author{
Gabriel Nshizirungu', Marco Mpimbo $^{2}$ and Vedaste Mutarutinya ${ }^{3}$ \\ ${ }^{I}$ Department of Mathematics, University of Dar es Salaam, Tanzania \\ E-mail: gabrielnshizirungu@gmail.com \\ ${ }^{2}$ Department of Mathematics, University of Dar es Salaam, Tanzania \\ E-mail: kmpimbo33@gmail.com \\ ${ }^{3}$ Department of Mathematics, Science and Physical Education, University of Rwanda, Rwanda \\ E-mail: mutavedik@gmail.com
}

Received 15 Jan 2021, Revised 8 Jul 2021, Accepted 22 Jul 2021, Published Aug 2021

DOI: https://dx.doi.org/10.4314/tjs.v47i3.21 inequality.

Keywords: Hardy's inequality, Integral inequalities, similar version, H $\ddot{~ l d e r ' s ~ i n e q u a l i t y, ~}$ Generalizations.

2010 Mathematics Subject Classification: 26D15.

\section{Introduction}

Hardy (1920) established his famous inequality for integrable functions as follows: if $p>1$ and $f$ is a non-negative $p$-integrable function on $(0, \infty)$, then $f(x)$ is integrable over the interval $(0, x)$ for each positive $x$ and

$$
\begin{aligned}
& \int_{0}^{\infty}\left(\frac{1}{x} \int_{0}^{x} f(t) d t\right)^{p} d x \\
& \leq\left(\frac{p}{p-1}\right)^{p} \int_{0}^{\infty} f^{p}(x) d x,
\end{aligned}
$$

where $\left(\frac{p}{p-1}\right)^{p}$ is the best possible constant. The Hardy inequality, from its origin and its subsequent development has attracted numerous mathematicians. From 1906 to 1928 , other mathematicians such as Landau, P'olya, Schur and Riesz were actively involved in the development of the Hardy inequality (Kufner et al. 2006). Since then, a lot of books and papers have been published in this area of research. Some of the notable literature in this area are the works due to Muckenhoupt (1972), Bradley (1978), Persson and Stepanov (2002),Wedestig
(2003), Persson (2018) and Ajisope and Rauf (2019).

Hardy-type integral inequalities have various practical applications in mathematics and related fields. Some of the applications are found in solvability of elliptic equations in Sobolev spaces, hydrodynamic problems, Partial differential equations and spectral gaps on trees.

An important inequality which we will use frequently in this paper is the Hölder's inequality and we present it as a lemma.

Lemma 1: Let $X$ be a measure space and let $p, q \in[1, \infty]$ where $\frac{1}{p}+\frac{1}{q}=1$. Then for all measurable real-(or complex) valued functions $f$ and $g$ on $X,\|f g\|_{1} \leq\|f\|_{p}\|g\|_{q}$.

Sulaiman (2012) presented and proved the following results similar to Hardy's inequality: Theorem 1: Let $f$ be a positive function defined on $[a, b] \subseteq(0, \infty)$ and define $F(x)=$ $\int_{a}^{x} f(t) d t$. Then,

1114 
(i) for $p \geq 1$,

$$
p \int_{a}^{b}\left(\frac{F(x)}{x}\right)^{p} d x \leq(b-a)^{p} \int_{a}^{b}\left(\frac{f(x)}{x}\right)^{p} d x-\int_{a}^{b}\left(1-\frac{a}{x}\right)^{p} f^{p}(x) d x .
$$

(ii) for $0<p<1$

$$
p \int_{a}^{b}\left(\frac{F(x)}{x}\right)^{p} d x \geq\left(1-\frac{a}{b}\right)^{p} \int_{a}^{b} f^{p}(x) d x-\frac{1}{b^{p}} \int_{a}^{b}(x-a)^{p} f^{p}(x) d x .
$$

Sroysang (2013) presented the generalizations of some integral inequalities similar to Hardy's inequality into the following two forms:

Theorem 2: Assume that $f>0$ defined on $[a, b] \subseteq(0, \infty)$ and $p \geq 1, q>0$. Define $F(x)=$ $\int_{a}^{x} f(t) d t$. Then

$$
p \int_{a}^{b} \frac{F^{p}(x)}{x^{q}} d x \leq(b-a)^{p} \int_{a}^{b} \frac{f^{p}(x)}{x^{q}} d x-\int_{a}^{b} \frac{(x-a)^{p}}{x^{q}} f^{p}(x) d x,
$$

and by considering the reverse integral inequality, the generalization is the following result:

Theorem 3: Assume that $f>0$ defined on $[a, b] \subseteq(0, \infty)$ and $0<p<1, q>0$. Define $F(x)=$ $\int_{a}^{x} f(t) d t$. Then

$$
p \int_{a}^{b} \frac{F^{p}(x)}{x^{q}} d x \geq \frac{(b-a)^{p}}{b^{p}} \int_{a}^{b} f^{p}(x) d x-\frac{1}{b^{p}} \int_{a}^{b}(x-a)^{p} f^{p}(x) d x .
$$

Wu and Sroysang (2015) established a further generalization of certain integral inequalities similar to Hardy's inequality into the following assertion:

Theorem 4: Let $p \geq 1$ and $q>0$. Assume that $f, g>0$ on $[a, b] \subseteq(0, \infty)$ such that $g$ is nondecreasing. Define $F(x)=\int_{a}^{x} f(t) d t$ for all $x>a$. Then, the inequality;

$$
p \int_{a}^{b} \frac{F^{p}(x)}{g^{q}(x)} d x \leq(b-a)^{p} \int_{a}^{b} \frac{f^{p}(x)}{g^{q}(x)} d x-\int_{a}^{b} \frac{(x-a)^{p}}{g^{q}(x)} f^{p}(x) d x .
$$

holds, and by considering the reverse integral inequalities similar to Hardy's inequality, Wu and Sroysang (2015) stated the following result:

Theorem 5: Let $0<p<1$ and $q>0$. Assume that $f, g>0$ on $[a, b] \subseteq(0, \infty)$ such that $g$ is non-decreasing. Define $F(x)=\int_{a}^{x} f(t) d t$ for all $x>a$. Then, the following inequality holds:

$$
p \int_{a}^{b} \frac{F^{p}(x)}{g^{q}(x)} d x \geq \frac{(b-a)^{p}}{g^{q}(b)} \int_{a}^{b} f^{p}(x) d x-\frac{1}{g^{q}(b)} \int_{a}^{b}(x-a)^{p} f^{p}(x) d x
$$

In this paper, the improvements and generalizations of both inequality (1) and inequality (2) are established by the use of Hölder's inequality, the reverse Hölder's inequality and other measure theoretical techniques.

\section{Main Results}

Theorem 6: Let $p \geq 1$ and $q>0$. Assume that $f, g>0$ on $[a, b] \subseteq(0, \infty)$ such that $g$ is nondecreasing. Define $F^{*}(x)=\int_{x}^{b} f(t) d t$ for all $b>x$. Then

$$
p \int_{a}^{b} \frac{\left(F^{*}\right)^{p}(x)}{g^{q}(x)} d x \leq \frac{(b-a)^{p}}{g^{q}(a)} \int_{a}^{b} f^{p}(x) d x-\frac{1}{g^{q}(a)} \int_{a}^{b}(b-x)^{p} f^{p}(x) d x .
$$

Proof. Since $g$ is non-decreasing and by the Hölder's inequality, we obtain that 


$$
\begin{gathered}
\int_{a}^{b} \frac{\left(F^{*}\right)^{p}(x)}{g^{q}(x)} d x=\int_{a}^{b} g^{-q}(x)\left(\int_{x}^{b} f(t) d t\right)^{p} d x \\
\leq \int_{a}^{b} g^{-q}(x)\left(\left(\int_{x}^{b} f^{p}(t) d t\right)^{\frac{1}{p}}\left(\int_{x}^{b} d t\right)^{\frac{p-1}{p}}\right)^{p} d x \\
=\int_{a}^{b} g^{-q}(x)\left(\left(\int_{x}^{b} f^{p}(t) d t\right)^{\frac{1}{p}}(b-x)^{\frac{p-1}{p}}\right) d x \\
=\int_{a}^{b} g^{-q}(x)\left(\int_{x}^{b} f^{p}(t) d t\right)(b-x)^{p-1} d x \\
=\int_{a}^{b} \int_{x}^{b} g^{-q}(x)(b-x)^{p-1} f^{p}(t) d t d x \\
=\int_{a}^{b} \int_{a}^{t} g^{-q}(x)(b-x)^{p-1} f^{p}(t) d x d t \\
\leq \int_{a}^{b} \int_{a}^{t} g^{-q}(a)(b-x)^{p-1} f^{p}(t) d x d t \\
=g^{-q}(a) \int_{a}^{b} f^{p}(t)\left(\int_{a}^{t}(b-x)^{p-1} d x\right) d t \\
=\frac{g^{-q}(a) \int_{a}^{b} f^{p}(t)\left(\frac{(b-a)^{p}-(b-t)^{p}}{p}\right) d t}{p}\left((b-a)^{p} \int_{a}^{b} f^{p}(t) d t-\int_{a}^{b}(b-t)^{p} f^{p}(t) d t\right)
\end{gathered}
$$

Corollary 1: Let $p \geq 1$, and assume that $f, g>0$ on $[a, b] \subseteq(0, \infty)$ such that $g$ is nondecreasing. Define $F^{*}(x)=\int_{x}^{b} f(t) d t$ for all $b>x$. Then

$$
p \int_{a}^{b}\left(\frac{F^{*}(x)}{g(x)}\right)^{p} d x \leq \frac{(b-a)^{p}}{g^{p}(a)} \int_{a}^{b} f^{p}(x) d x-\frac{1}{g^{p}(a)} \int_{a}^{b}(b-x)^{p} f^{p}(x) d x .
$$

Proof. Since $g$ is non-decreasing and by the Hölder's inequality, we have that

$$
\begin{gathered}
\int_{a}^{b}\left(\frac{F^{*}(x)}{g(x)}\right)^{p} d x=\int_{a}^{b} g^{-p}(x)\left(\int_{x}^{b} f(t) d t\right)^{p} d x \\
\leq \int_{a}^{b} g^{-p}(x)\left(\left(\int_{x}^{b} f^{p}(t) d t\right)^{\frac{1}{p}}\left(\int_{x}^{b} d t\right)^{\frac{p-1}{p}}\right)^{p} d x \\
=\int_{a}^{b} g^{-p}(x)\left(\left(\int_{x}^{b} f^{p}(t) d t\right)^{\frac{1}{p}}(b-x)^{\frac{p-1}{p}}\right)^{p} d x \\
=\int_{a}^{b} g^{-p}(x)\left(\int_{x}^{b} f^{p}(t) d t\right)(b-x)^{p-1} d x \\
=\int_{a}^{b} \int_{x}^{b} g^{-p}(x)(b-x)^{p-1} f^{p}(t) d t d x
\end{gathered}
$$




$$
\begin{gathered}
=\int_{a}^{b} \int_{a}^{t} g^{-p}(x)(b-x)^{p-1} f^{p}(t) d x d t \\
\leq \int_{a}^{b} \int_{a}^{t} g^{-p}(a)(b-x)^{p-1} f^{p}(t) d x d t \\
=g^{-p}(a) \int_{a}^{b} f^{p}(t)\left(\int_{a}^{t}(b-x)^{p-1} d x\right) d t \\
=g^{-p}(a) \int_{a}^{b} f^{p}(t)\left(\frac{(b-a)^{p}-(b-t)^{p}}{p}\right) d t \\
=\frac{g^{-p}(a)}{p}\left((b-a)^{p} \int_{a}^{b} f^{p}(t) d t-\int_{a}^{b}(b-t)^{p} f^{p}(t) d t\right)
\end{gathered}
$$

Corollary 2: Let $p \geq 1$, and assume that $f>0$ on $[a, b] \subseteq(0, \infty)$. Define $F^{*}(x)=\int_{x}^{b} f(t) d t$ for all $b>x$. Then

$$
p \int_{a}^{b}\left(\frac{F^{*}(x)}{x}\right)^{p} d x \leq\left(\frac{b}{a}-1\right)^{p} \int_{a}^{b} f^{p}(x) d x-\frac{1}{a^{p}} \int_{a}^{b}(b-x)^{p} f^{p}(x) d x .
$$

Proof. Using the Hölder's inequality, one has,

$$
\begin{gathered}
\int_{a}^{b}\left(\frac{F^{*}(x)}{x}\right)^{p} d x=\int_{a}^{b} x^{-p}\left(\int_{x}^{b} f(t) d t\right)^{p} d x \\
\leq \int_{a}^{b} x^{-p}\left(\left(\int_{x}^{b} f^{p}(t) d t\right)^{\frac{1}{p}}\left(\int_{x}^{b} d t\right)^{\frac{p-1}{p}}\right)_{p}^{p} d x \\
=\int_{a}^{b} x^{-p}\left(\left(\int_{x}^{b} f^{p}(t) d t\right)^{\frac{1}{p}}(b-x)^{\frac{p-1}{p}}\right)^{p} d x \\
=\int_{a}^{b} x^{-p}\left(\int_{x}^{b} f^{p}(t) d t\right)(b-x)^{p-1} d x \\
=\int_{a}^{b} \int_{x}^{b} x^{-p}(b-x)^{p-1} f^{p}(t) d t d x=\int_{a}^{b} \int_{a}^{t} x^{-p}(b-x)^{p-1} f^{p}(t) d x d t \\
\leq \int_{a}^{b} \int_{a}^{t} a^{-p}(b-x)^{p-1} f^{p}(t) d x d t=a^{-p} \int_{a}^{b} f^{p}(t)\left(\int_{a}^{t}(b-x)^{p-1} d x\right) d t \\
=a^{-p} \int_{a}^{b} f^{p}(t)\left(\frac{(b-a)^{p}-(b-t)^{p}}{p}\right) d t \\
=\frac{a^{-p}}{p}\left((b-a)^{p} \int_{a}^{b} f^{p}(t) d t-\int_{a}^{b}(b-t)^{p} f^{p}(t) d t\right) \\
=\frac{1}{p}\left(\left(\frac{b}{a}-1\right)^{p} \int_{a}^{b} f^{p}(t) d t-\frac{1}{a^{p}} \int_{a}^{b}(b-x)^{p} f^{p}(t) d t .\right) \#
\end{gathered}
$$

Corollary 3: Let $p \geq 1$ and $q>0$. Assume that $f>0$ on $[a, b] \subseteq(0, \infty)$. Define $F^{*}(x)=$ $\int_{x}^{b} f(t) d t$ for all $b>x$. Then

$$
p \int_{a}^{b} \frac{\left(F^{*}\right)^{p}(x)}{(b-x)^{q}} d x \leq(b-a)^{p-q} \int_{a}^{b} f^{p}(x) d x-\frac{1}{(b-a)^{q}} \int_{a}^{b}(b-x)^{p} f^{p}(x) d x .
$$


Proof. Using the Hölder's inequality, we obtain that

$$
\begin{aligned}
& \int_{a}^{b} \frac{\left(F^{*}\right)^{p}(x)}{(b-x)^{q}} d x=\int_{a}^{b}(b-x)^{-q}\left(\int_{x}^{b} f(t) d t\right)^{p} d x \\
& \leq \int_{a}^{b}(b-x)^{-q}\left(\left(\int_{x}^{b} f^{p}(t) d t\right)^{\frac{1}{p}}\left(\int_{x}^{b} d t\right)^{\frac{p-1}{p}}\right)^{p} d x \\
& =\int_{a}^{b}(b-x)^{-q}\left(\left(\int_{x}^{b} f^{p}(t) d t\right)^{\frac{1}{p}}(b-x)^{\frac{p-1}{p}}\right)^{p} d x \\
& =\int_{a}^{b}(b-x)^{-q}\left(\int_{x}^{b} f^{p}(t) d t\right)(b-x)^{p-1} d \\
& =\int_{a}^{b} \int_{x}^{b}(b-x)^{-q}(b-x)^{p-1} f^{p}(t) d t d x \\
& =\int_{a}^{b} \int_{a}^{t}(b-x)^{-q}(b-x)^{p-1} f^{p}(t) d x d t \\
& \leq \int_{a}^{b} \int_{a}^{t}(b-a)^{-q}(b-x)^{p-1} f^{p}(t) d x d t \\
& =(b-a)^{-q} \int_{a}^{b} f^{p}(t)\left(\int_{a}^{t}(b-x)^{p-1} d x\right) d t \\
& =(b-a)^{-q} \int_{a}^{b} f^{p}(t)\left(\frac{(b-a)^{p}-(b-t)^{p}}{p}\right) d t \\
& =\frac{(b-a)^{-q}}{p}\left((b-a)^{p} \int_{a}^{b} f^{p}(t) d t-\int_{a}^{b}(b-t)^{p} f^{p}(t) d t\right) \#
\end{aligned}
$$

Corollary 4: Let $p \geq 1$ and assume that $f>0$ on $[a, b] \subseteq(0, \infty)$. Define $F^{*}(x)=\int_{x}^{b} f(t) d t$ for all $b>x$. Then

$$
p \int_{a}^{b}\left(\frac{F^{*}(x)}{b-x}\right)^{p} d x \leq \int_{a}^{b} f^{p}(x) d x-\frac{1}{(b-a)^{p}} \int_{a}^{b}(b-x)^{p} f^{p}(x) d x .
$$

Proof. The Hölder's inequality gives,

$$
\begin{gathered}
\int_{a}^{b}\left(\frac{F^{*}(x)}{b-x}\right)^{p} d x=\int_{a}^{b}(b-x)^{-p}\left(\int_{x}^{b} f(t) d t\right)^{p} d x \\
\leq \int_{a}^{b}(b-x)^{-p}\left(\left(\int_{x}^{b} f^{p}(t) d t\right)^{\frac{1}{p}}\left(\int_{x}^{b} d t\right)^{\frac{p-1}{p}}\right)^{p} d x \\
=\int_{a}^{b}(b-x)^{-p}\left(\left(\int_{x}^{b} f^{p}(t) d t\right)^{\frac{1}{p}}(b-x)^{\frac{p-1}{p}}\right)^{p} d x \\
=\int_{a}^{b}(b-x)^{-p}\left(\int_{x}^{b} f^{p}(t) d t\right)(b-x)^{p-1} d x \\
=\int_{a}^{b} \int_{x}^{b}(b-x)^{-p}(b-x)^{p-1} f^{p}(t) d t d x
\end{gathered}
$$




$$
\begin{gathered}
=\int_{a}^{b} \int_{a}^{t}(b-x)^{-p}(b-x)^{p-1} f^{p}(t) d x d t \\
\leq \int_{a}^{b} \int_{a}^{t}(b-a)^{-p}(b-x)^{p-1} f^{p}(t) d x d t \\
=(b-a)^{-p} \int_{a}^{b} f^{p}(t)\left(\int_{a}^{t}(b-x)^{p-1} d x\right) d t \\
=(b-a)^{-p} \int_{a}^{b} f^{p}(t)\left(\frac{(b-a)^{p}-(b-t)^{p}}{p}\right) d t \\
=\frac{(b-a)^{-p}}{p}\left((b-a)^{p} \int_{a}^{b} f^{p}(t) d t-\int_{a}^{b}(b-t)^{p} f^{p}(t) d t\right) \\
=\frac{1}{p}\left(\int_{a}^{b} f^{p}(t) d t-\frac{1}{(b-a)^{p}} \int_{a}^{b}(b-t)^{p} f^{p}(t) d t\right)
\end{gathered}
$$

Corollary 5: Let $p \geq 1$ and $q>0$. Assume that $f>0$ on $[a, b] \subseteq(0, \infty)$. Define $F^{*}(x)=$ $\int_{x}^{b} f(t) d t$ for all $b>x$. Then

$$
p \int_{a}^{b} \frac{\left(F^{*}\right)^{p}(x)}{x^{q}} d x \leq \frac{(b-a)^{p}}{a^{q}} \int_{a}^{b} f^{p}(x) d x-\frac{1}{a^{q}} \int_{a}^{b}(b-x)^{p} f^{p}(x) d x .
$$

Proof. From the Hölder's inequality, we have,

$$
\begin{gathered}
\int_{a}^{b} \frac{\left(F^{*}\right)^{p}(x)}{x^{q}} d x=\int_{a}^{b} x^{-q}\left(\int_{x}^{b} f(t) d t\right)^{p} d x \\
\leq \int_{a}^{b} x^{-q}\left(\left(\int_{x}^{b} f^{p}(t) d t\right)^{\frac{1}{p}}\left(\int_{x}^{b} d t\right)^{\frac{p-1}{p}}\right)^{p} d x \\
=\int_{a}^{b} x^{-q}\left(\left(\int_{x}^{b} f^{p}(t) d t\right)^{\frac{1}{p}}(b-x)^{\frac{p-1}{p}}\right)^{p} d x \\
=\int_{a}^{b} x^{-q}\left(\int_{x}^{b} f^{p}(t) d t\right)(b-x)^{p-1} d x \\
=\int_{a}^{b} \int_{x}^{b} x^{-q}(b-x)^{p-1} f^{p}(t) d t d x \\
=\int_{a}^{b} \int_{a}^{t} x^{-q}(b-x)^{p-1} f^{p}(t) d x d t \\
\leq \int_{a}^{b} \int_{a}^{t} a^{-q}(b-x)^{p-1} f^{p}(t) d x d t \\
=a^{-q} \int_{a}^{b} f^{p}(t)\left(\int_{a}^{t}(b-x)^{p-1} d x\right) d t \\
=a^{-q} \int_{a}^{b} f^{p}(t)\left(\frac{(b-a)^{p}-(b-t)^{p}}{p}\right) d t \\
=\frac{a^{-q}}{p}\left((b-a)^{p} \int_{a}^{b} f^{p}(t) d t-\int_{a}^{b}(b-t)^{p} f^{p}(t) d t\right) \#
\end{gathered}
$$


Theorem 7: Let $0<p<1$ and $q>0$. Assume that $f, g>0$ on $[a, b] \subseteq(0, \infty)$ such that $g$ is non-decreasing. Define $F^{*}(x)=\int_{x}^{b} f(t) d t$ for all $b>x$. Then

$$
p \int_{a}^{b} \frac{\left(F^{*}\right)^{p}(x)}{g^{q}(x)} d x \geq(b-a)^{p} \int_{a}^{b} \frac{f^{p}(x)}{g^{q}(x)} d x-\int_{a}^{b} \frac{(b-x)^{p}}{g^{q}(x)} f^{p}(x) d x .
$$

Proof. Since $g$ is non-decreasing and by the use of reverse Hölder's inequality, we obtain that

$$
\begin{gathered}
\int_{a}^{b} \frac{\left(F^{*}\right)^{p}(x)}{g^{q}(x)} d x=\int_{a}^{b} g^{-q}(x)\left(\int_{x}^{b} f(t) d t\right)^{p} d x \\
\geq \int_{a}^{b} g^{-q}(x)\left(\left(\int_{x}^{b} f^{p}(t) d t\right)^{\frac{1}{p}}\left(\int_{x}^{b} d t\right)^{\frac{p-1}{p}}\right)^{p} d x \\
=\int_{a}^{b} g^{-q}(x)\left(\left(\int_{x}^{b} f^{p}(t) d t\right)^{\frac{1}{p}}(b-x)^{\frac{p-1}{p}}\right) d x \\
=\int_{a}^{b} g^{-q}(x)\left(\int_{x}^{b} f^{p}(t) d t\right)(b-x)^{p-1} d x \\
=\int_{a}^{b} \int_{x}^{b} g^{-q}(x)(b-x)^{p-1} f^{p}(t) d t d x \\
=\int_{a}^{b} \int_{a}^{t} g^{-q}(x)(b-x)^{p-1} f^{p}(t) d x d t \\
\geq \int_{a}^{b} \int_{a}^{t} g^{-q}(t)(b-x)^{p-1} f^{p}(t) d x d t \\
=\int_{a}^{b} g^{-q}(t) f^{p}(t)\left(\int_{a}^{t}(b-x)^{p-1} d x\right) d t \\
=\int_{a}^{b} g^{-q}(t) f^{p}(t)\left(\frac{(b-a)^{p}-(b-t)^{p}}{p}\right) d t \\
\left.(b-a)^{p} \int_{a}^{b} \frac{f^{p}(t)}{g^{q}(t)} d t-\int_{a}^{b} \frac{(b-t)^{p}}{g^{q}(t)} f^{p}(t) d t\right)
\end{gathered}
$$

Corollary 6: Let $0<p<1$ and assume that $f, g>0$ on $[a, b] \subseteq(0, \infty)$ such that $g$ is nondecreasing. Define $F^{*}(x)=\int_{x}^{b} f(t) d t$ for all $b>x$. Then

$$
p \int_{a}^{b}\left(\frac{F^{*}(x)}{g(x)}\right)^{p} d x \geq(b-a)^{p} \int_{a}^{b}\left(\frac{f(x)}{g(x)}\right)^{p} d x-\int_{a}^{b}\left(\frac{b-x}{g(x)}\right)^{p} f^{p}(x) d x .
$$

Proof. Since $g$ is non-decreasing and by the use of reverse Hölder's inequality, we obtain that

$$
\begin{aligned}
& \int_{a}^{b}\left(\frac{F^{*}(x)}{g(x)}\right)^{p} d x=\int_{a}^{b} g^{-p}(x)\left(\int_{x}^{b} f(t) d t\right)^{p} d x \\
& \geq \int_{a}^{b} g^{-p}(x)\left(\left(\int_{x}^{b} f^{p}(t) d t\right)^{\frac{1}{p}}\left(\int_{x}^{b} d t\right)^{\frac{p-1}{p}}\right)^{p} d x \\
& =\int_{a}^{b} g^{-p}(x)\left(\left(\int_{x}^{b} f^{p}(t) d t\right)^{\frac{1}{p}}(b-x)^{\frac{p-1}{p}}\right)^{p} d x
\end{aligned}
$$




$$
\begin{gathered}
=\int_{a}^{b} g^{-p}(x)\left(\int_{x}^{b} f^{p}(t) d t\right)(b-x)^{p-1} d x \\
=\int_{a}^{b} \int_{x}^{b} g^{-p}(x)(b-x)^{p-1} f^{p}(t) d t d x \\
=\int_{a}^{b} \int_{a}^{t} g^{-p}(x)(b-x)^{p-1} f^{p}(t) d x d t \\
\geq \int_{a}^{b} \int_{a}^{t} g^{-p}(t)(b-x)^{p-1} f^{p}(t) d x d t \\
=\int_{a}^{b} g^{-p}(t) f^{p}(t)\left(\int_{a}^{t}(b-x)^{p-1} d x\right) d t \\
=\int_{a}^{b} g^{-p}(t) f^{p}(t)\left(\frac{(b-a)^{p}-(b-t)^{p}}{p}\right) d t \\
=\frac{1}{p}\left((b-a)^{p} \int_{a}^{b}\left(\frac{f(t)}{g(t)}\right)^{p} d t-\int_{a}^{b}\left(\frac{b-t}{g(t)}\right)^{p} f^{p}(t) d t\right)
\end{gathered}
$$

Corollary 7: Let $0<p<1$ and assume that $f>0$ on $[a, b] \subseteq(0, \infty)$. Define $F^{*}(x)=\int_{x}^{b} f(t) d t$ for all $b>x$. Then

$$
p \int_{a}^{b}\left(\frac{F^{*}(x)}{x}\right)^{p} d x \geq(b-a)^{p} \int_{a}^{b}\left(\frac{f(x)}{x}\right)^{p} d x-\int_{a}^{b}\left(\frac{b-x}{x}\right)^{p} f^{p}(x) d x .
$$

Proof. Making use of the reverse Hölder's inequality, we observe that

$$
\begin{gathered}
\int_{a}^{b}\left(\frac{F^{*}(x)}{x}\right)^{p} d x=\int_{a}^{b} x^{-p}\left(\int_{x}^{b} f(t) d t\right)^{p} d x \\
\geq \int_{a}^{b} x^{-p}\left(\left(\int_{x}^{b} f^{p}(t) d t\right)^{\frac{1}{p}}\left(\int_{x}^{b} d t\right)^{\frac{p-1}{p}}\right)^{p} d x \\
=\int_{a}^{b} x^{-p}\left(\left(\int_{x}^{b} f^{p}(t) d t\right)^{\frac{1}{p}}(b-x)^{\frac{p-1}{p}}\right) d x \\
=\int_{a}^{b} x^{-p}\left(\int_{x}^{b} f^{p}(t) d t\right)(b-x)^{p-1} d x \\
=\int_{a}^{b} \int_{x}^{b} x^{-p}(b-x)^{p-1} f^{p}(t) d t d x \\
=\int_{a}^{b} \int_{a}^{t} x^{-p}(b-x)^{p-1} f^{p}(t) d x d t \\
\geq \int_{a}^{b} \int_{a}^{t} t^{-p}(b-x)^{p-1} f^{p}(t) d x d t \\
=\int_{a}^{b} t^{-p} f^{p}(t)\left(\int_{a}^{t}(b-x)^{p-1} d x\right) d t \\
=\int_{a}^{b} t^{-p} f^{p}(t)\left(\frac{(b-a)^{p}-(b-t)^{p}}{p}\right) d t
\end{gathered}
$$




$$
=\frac{1}{p}\left((b-a)^{p} \int_{a}^{b}\left(\frac{f(t)}{t}\right)^{p} d t-\int_{a}^{b}\left(\frac{b-t}{t}\right)^{p} f^{p}(t) d t\right) \#
$$

Corollary 8: Let $0<p<1$ and $q>0$. Assume that $f>0$ on $[a, b] \subseteq(0, \infty)$. Define $F^{*}(x)=$ $\int_{x}^{b} f(t) d t$ for all $b>x$. Then

$$
p \int_{a}^{b} \frac{\left(F^{*}\right)^{p}(x)}{(b-x)^{q}} d x \geq(b-a)^{p} \int_{a}^{b} \frac{f^{p}(x)}{(b-x)^{q}} d x-\int_{a}^{b}(b-x)^{p-q} f^{p}(x) d x .
$$

Proof. By the use of reverse Hölder's inequality, we obtain that

$$
\begin{gathered}
\int_{a}^{b} \frac{\left(F^{*}\right)^{p}(x)}{(b-x)^{q}} d x=\int_{a}^{b}(b-x)^{-q}\left(\int_{x}^{b} f(t) d t\right)^{p} d x \\
\geq \int_{a}^{b}(b-x)^{-q}\left(\left(\int_{x}^{b} f^{p}(t) d t\right)^{\frac{1}{p}}\left(\int_{x}^{b} d t\right)^{\frac{p-1}{p}}\right)^{p} d x \\
=\int_{a}^{b}(b-x)^{-q}\left(\left(\int_{x}^{b} f^{p}(t) d t\right)^{\frac{1}{p}}(b-x)^{\frac{p-1}{p}}\right) d x \\
=\int_{a}^{b}(b-x)^{-q}\left(\int_{x}^{b} f^{p}(t) d t\right)(b-x)^{p-1} d x \\
=\int_{a}^{b} \int_{x}^{b}(b-x)^{-q}(b-x)^{p-1} f^{p}(t) d t d x \\
=\int_{a}^{b} \int_{a}^{t}(b-x)^{-q}(b-x)^{p-1} f^{p}(t) d x d t \\
\geq \int_{a}^{b} \int_{a}^{t}(b-t)^{-q}(b-x)^{p-1} f^{p}(t) d x d t \\
=\int_{a}^{b}(b-t)^{-q} f^{p}(t)\left(\int_{a}^{t}(b-x)^{p-1} d x\right) d t \\
=\int_{a}^{b}(b-t)^{-q} f^{p}(t)\left(\frac{(b-a)^{p}-(b-t)^{p}}{p}\right) d t \\
\left.(b-a)^{p} \int_{a}^{b} \frac{f^{p}(t)}{(b-t)^{q}} d t-\int_{a}^{b}(b-t)^{p-q} f^{p}(t) d t\right)
\end{gathered}
$$

Corollary 9: Let $0<p<1$ and assume that $f>0$ on $[a, b] \subseteq(0, \infty)$. Define $F^{*}(x)=\int_{x}^{b} f(t) d t$ for all $b>x$. Then

$$
p \int_{a}^{b}\left(\frac{F^{*}(x)}{b-x}\right)^{p} d x \geq(b-a)^{p} \int_{a}^{b}\left(\frac{f(x)}{b-x}\right)^{p} d x-\int_{a}^{b} f^{p}(x) d x .
$$

Proof. By the use of the reverse Hölder's inequality, we obtain that

$$
\begin{aligned}
& \int_{a}^{b}\left(\frac{F^{*}(x)}{b-x}\right)^{p} d x=\int_{a}^{b}(b-x)^{-p}\left(\int_{x}^{b} f(t) d t\right)^{p} d x \\
& \geq \int_{a}^{b}(b-x)^{-p}\left(\left(\int_{x}^{b} f^{p}(t) d t\right)^{\frac{1}{p}}\left(\int_{x}^{b} d t\right)^{\frac{p-1}{p}}\right)^{p} d x
\end{aligned}
$$




$$
\begin{gathered}
=\int_{a}^{b}(b-x)^{-p}\left(\left(\int_{x}^{b} f^{p}(t) d t\right)^{\frac{1}{p}}(b-x)^{\frac{p-1}{p}}\right)^{p} d x \\
=\int_{a}^{b}(b-x)^{-p}\left(\int_{x}^{b} f^{p}(t) d t\right)(b-x)^{p-1} d x \\
=\int_{a}^{b} \int_{x}^{b}(b-x)^{-p}(b-x)^{p-1} f^{p}(t) d t d x \\
=\int_{a}^{b} \int_{a}^{t}(b-x)^{-p}(b-x)^{p-1} f^{p}(t) d x d t \\
\geq \int_{a}^{b} \int_{a}^{t}(b-t)^{-p}(b-x)^{p-1} f^{p}(t) d x d t \\
=\int_{a}^{b}(b-t)^{-p} f^{p}(t)\left(\int_{a}^{t}(b-x)^{p-1} d x\right) d t \\
=\int_{a}^{b}(b-t)^{-p} f^{p}(t)\left(\frac{(b-a)^{p}-(b-t)^{p}}{p}\right) d t \\
=\frac{1}{p}\left((b-a)^{p} \int_{a}^{b}\left(\frac{f(t)}{b-t}\right)^{p} d t-\int_{a}^{b} f^{p}(t) d t\right) \#
\end{gathered}
$$

Corollary 10: Let $0<p<1$ and $q>0$. Assume that $f>0$ on $[a, b] \subseteq(0, \infty)$. Define $F^{*}(x)=$ $\int_{x}^{b} f(t) d t$ for all $b>x$. Then

$$
p \int_{a}^{b} \frac{\left(F^{*}\right)^{p}(x)}{x^{q}} d x \geq(b-a)^{p} \int_{a}^{b} \frac{f^{p}(x)}{x^{q}} d x-\int_{a}^{b} \frac{(b-x)^{p}}{x^{q}} f^{p}(x) d x .
$$

Proof. By the use of reverse Hölder's inequality, we obtain that

$$
\begin{gathered}
\int_{a}^{b} \frac{\left(F^{*}\right)^{p}(x)}{x^{q}} d x=\int_{a}^{b} x^{-q}\left(\int_{x}^{b} f(t) d t\right)^{p} d x \\
\geq \int_{a}^{b} x^{-q}\left(\left(\int_{x}^{b} f^{p}(t) d t\right)^{\frac{1}{p}}\left(\int_{x}^{b} d t\right)^{\frac{p-1}{p}}\right)^{p} d x \\
=\int_{a}^{b} x^{-q}\left(\left(\int_{x}^{b} f^{p}(t) d t\right)^{\frac{1}{p}}(b-x)^{\frac{p-1}{p}}\right)^{p} d x \\
=\int_{a}^{b} x^{-q}\left(\int_{x}^{b} f^{p}(t) d t\right)(b-x)^{p-1} d x \\
=\int_{a}^{b} \int_{x}^{b} x^{-q}(b-x)^{p-1} f^{p}(t) d t d x \\
=\int_{a}^{b} \int_{a}^{t} x^{-q}(b-x)^{p-1} f^{p}(t) d x d t \\
\geq \int_{a}^{b} \int_{a}^{t} t^{-q}(b-x)^{p-1} f^{p}(t) d x d t
\end{gathered}
$$




$$
\begin{gathered}
=\int_{a}^{b} t^{-q} f^{p}(t)\left(\int_{a}^{t}(b-x)^{p-1} d x\right) d t \\
=\int_{a}^{b} t^{-q} f^{p}(t)\left(\frac{(b-a)^{p}-(b-t)^{p}}{p}\right) d t \\
=\frac{1}{p}\left((b-a)^{p} \int_{a}^{b} \frac{f^{p}(t)}{t^{q}} d t-\int_{a}^{b} \frac{(b-t)^{p}}{t^{q}} f^{p}(t) d t\right)
\end{gathered}
$$

\section{Conclusion}

In this paper, we managed to generalize the integral inequalities similar to Hardy's inequality. In so doing, we have answered the open problem stated in $\mathrm{Wu}$ and Sroysang (2015).

\section{References}

Ajisope MO Rauf K 2019 On some refinements of Hardy-type Integral inequalities. Int. J. Math. Comput. 14(4): 879-888.

Bradley JS 1978 Hardy inequalities with mixed norms. Canad. Math. Bull. 21: 405-408.

Hardy GH 1920 Notes on a theorem of Hilbert. Math. Z. 6: 314-317.

Kufner A, Maligranda L and Persson LE 2006 The prehistory of the Hardy inequality. Am. Math. Mon. 113: 715-732.

Muckenhoupt B 1972 Hardy's inequality with weights. Studia Math. 44: 31-38.

Persson LE 2018 The Hardy inequality: Prehistory, history and current status. Luleå University of Technology, Sweden, and
UiT, The Arctic University of Norway. 156.

Persson LE and Stepanov V 2002 Weighted integral inequalities with the geometric mean operator. J. Inequal. Appl. 7: 727746.

Sroysang B 2013 A generalization of some integral inequalities similar to Hardy's inequality. Math. Aeterna. 3(7): 593-596.

Sulaiman WT 2012 Reverses of Minkowski's, Hölder's, and Hardy's Inequalities. Int. J. Mod. Math. Sci. 1(1): 14-24.

Wedestig A 2013 Weighted inequalities of Hardy-types and their limiting inequalities. $\mathrm{PhD}$ thesis, Department of Mathematics, Luleå University of Technology.

Wu S and Sroysang B 2015 A further Generalization of certain integral inequalities similar to Hardy's inequality. Longyan University, Fujian, 364012, P.R. China and Thammasat University, Pathumthan, 12121, Thailand. 1-11. 